\title{
Metastatic Breast Cancer Presenting as Air-space Consolidation on Chest Computed Tomography
}

\author{
Hiroshi Ohnishi, Yoshinori Haruta, Akihito Yokoyama, Taku Nakashima, Noboru Hattori \\ and Nobuoki Kohno
}

\begin{abstract}
A 56-year-old woman suffered from hepatic and bone metastases of breast cancer. Two months after starting combination chemotherapy with trastuzumab and docetaxel, air-space consolidation was observed in the right lower lung lobe on a chest computed tomography (CT) and a high serum KL-6 level was detected. Drug-induced pneumonitis with organizing pneumonia type was suspected, however, a transbronchial lung biopsy and cytological examination of the bronchoalveolar lavage fluid provided evidence of metastatic breast cancer. While the lung is a frequently affected site from metastasis of breast cancer, we report a rare case presenting as air-space consolidation on a chest CT.
\end{abstract}

Key words: breast cancer, bronchioloalveolar carcinoma, capecitabine, human epidermal growth factor receptor type 2, mucinous carcinoma

(Inter Med 48: 727-731, 2009)

(DOI: 10.2169/internalmedicine.48.1849)

\section{Introduction}

Breast cancer is the major cancer found in women. Patients with breast cancer may show various presentations in chest radiographic images resulting from either cancer recurrence or therapy-related complications. The lung is a common site from metastasis of breast cancer with solitary or multiple pulmonary nodules, lymphangitic metastasis, and/or endobronchial metastasis being common manifestations (13). However, involvement as air-space consolidation is rare (1-3). Here, we report a case of breast cancer which metastasized to the lung and presented as air-space consolidation on chest computed tomography (CT).

\section{Case Report}

A 56-year-old woman was admitted to Hiroshima University Hospital for the evaluation of dry cough and abnormal opacity on chest radiography (Fig. 1A). The patient, a nonsmoker, had a history of breast cancer which was treated by a modified radical left mastectomy 5 years previously. The pathological diagnosis was stage $\mathrm{II}_{\mathrm{A}}\left(\mathrm{pT}_{2} \mathrm{~N}_{0} \mathrm{M}_{0}\right)$ breast cancer with invasive mucinous carcinoma mixed with papillotubular carcinoma (histological grade I). Four years after the mastectomy, multiple metastases of the breast cancer were found in the left supraclavicular lymph nodes, bones, and liver. The patient received combination chemotherapy with trastuzumab and docetaxel. Two months after the introduction of the combination chemotherapy, air-space consolidation mixed with ground-glass opacity gradually appeared in the right lower lung lobe on chest radiography and CT scan (Fig. 1A, 1B). Laboratory data revealed a highly elevated serum KL-6 level $(4,227 \mathrm{U} / \mathrm{mL}$, normal value $<500 \mathrm{U} / \mathrm{mL})$. Drug-induced pneumonitis with either organizing pneumonia (OP) or eosinophilic pneumonia (EP) type was suspected at first, since the appearance of opacity in the lung followed the introduction of combination chemotherapy.

Fine crackles in the lower posterior portion of the right hemithorax were heard on auscultation of the chest. Chest radiography and CT scan on admission showed a post-left mastectomy in addition to non-segmental air-space consolidation in the right lower lung field (Fig. 1A, 1B). Blood tests revealed increased serum level of not only KL-6 (4,400 $\mathrm{U} / \mathrm{mL})$, but CA15-3 (663 U/mL), carcinoembryonic antigen (CEA, 1,965.7 ng/mL), and cytokeratin-19 fragments (CY- 
A

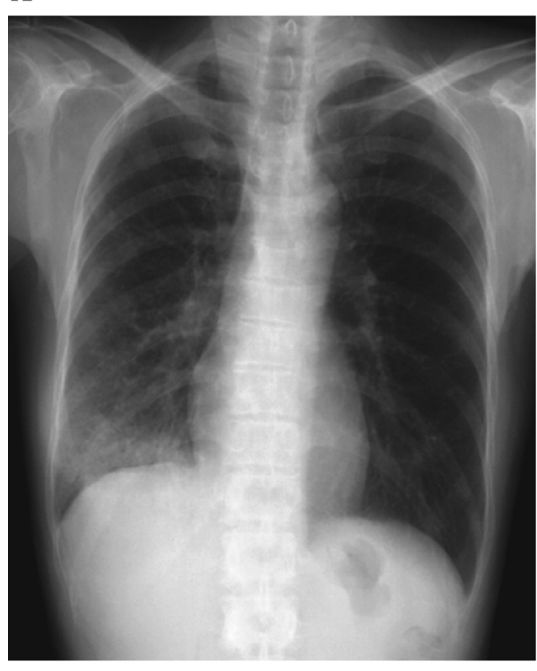

B

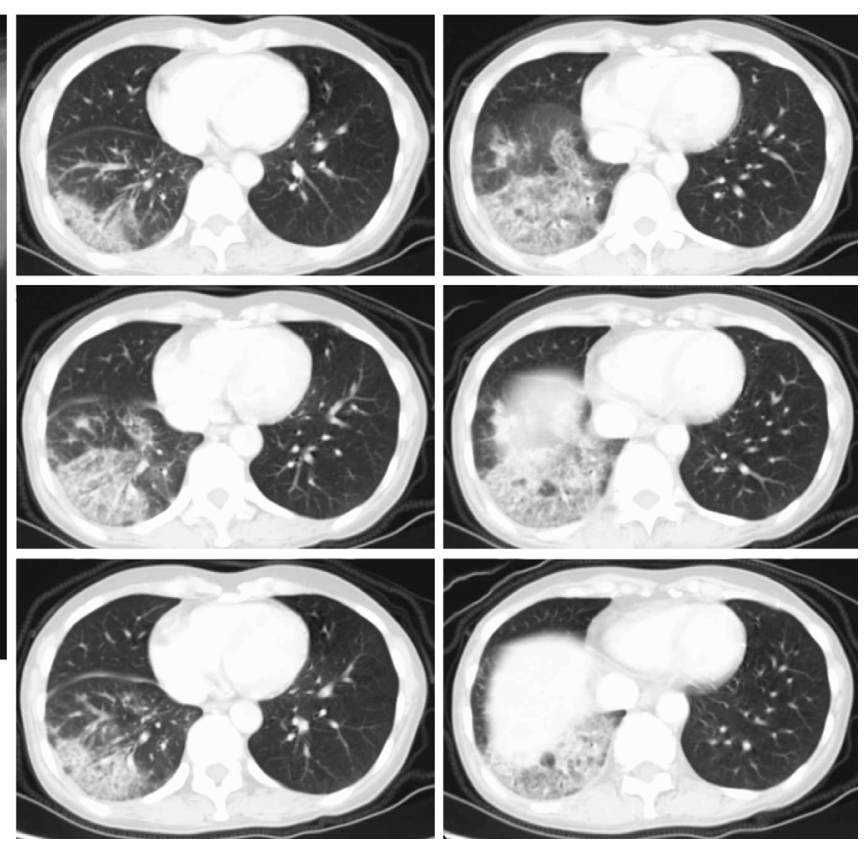

Figure 1. (A) Chest radiography on admission showed post-left mastectomy condition and consolidation opacity in the right lower lung field. (B) Chest CT scan on admission revealed non-segmental air-space consolidation mixed with ground-glass opacity in the right lower lung lobe.

FRA, $14.8 \mathrm{ng} / \mathrm{mL}$ ) as well. Serum levels of C-reactive protein $(0.3 \mathrm{mg} / \mathrm{dL})$ and peripheral blood eosinophil numbers $\left(1.8 \%\right.$, white blood cells count was $\left.5.03 \times 10^{3} / \mu \mathrm{L}\right)$ were found to be normal on admission.

Bronchoalveolar lavage (BAL) fluid from the right lower lung lobe $\left(\mathrm{S}^{10} \mathrm{~b}\right)$ revealed normal bacterial flora and significantly increased levels of KL-6 (1,190 U/mL), CA15-3 (47.1 U/mL), and CEA $(84.2 \mathrm{ng} / \mathrm{mL})$. Cytological examination of BAL fluid showed clusters of atypical vacuolated malignant cells suggesting adenocarcinoma. BAL fluid contained some neutrophils and lymphocytes, but no eosinophils. A transbronchial lung biopsy specimen from right lower lung lobe $\left(\mathrm{S}^{9} \mathrm{a}\right)$ proved to be histologically similar to the original left breast cancer specimen, a finding consistent with metastasis of the breast cancer (Fig. 2). Positive immunohistochemical staining of the lung biopsy for estrogen receptor (ER) and human epidermal growth factor receptor type 2 (Her2) (Her2 score; $3+$ ), which were also positive in the original left breast cancer specimen (Her2 score; 2+), provided further confirmation that the tumor originated in the breast. Whereas negative staining for the surfactant protein-A (SP-A) excluded the possibility that the origin of tumor was the lungs. These data strongly suggest that the air-space consolidation observed in the right lower lung lobe was the manifestation of metastatic breast cancer. Immunohistochemical staining for progesterone receptor was positive for the primary breast cancer specimen but not for the transbronchial lung biopsy specimen.

Since combination chemotherapy with trastuzumab and docetaxel had failed, the patient was treated with capecitabine monotherapy. Serum levels of CEA were decreased from $2,534.4 \mathrm{ng} / \mathrm{mL}$ to $1,768.8 \mathrm{ng} / \mathrm{mL}$ by capecitabine monotherapy (Fig. 3). Although multiple hepatic and bone metastatic lesions still remained, the lung metastatic lesion, showing as air-space consolidation, was dramatically decreased in size and almost undetectable on chest radiography and CT scan three months after receiving capecitabine monotherapy (Fig. 3).

\section{Discussion}

Breast cancer is the most prevalent type of cancer and second leading cause of cancer-related deaths in women in the U.S. (4). The incidence of breast cancer is lower in Japan than in U.S. or Western Europe, but is gradually increasing due to westernization of life style and diet.

Breast cancer is known to metastasize most frequently to the lung, but its involvement in air-space consolidation is extremely rare (1-3). Differential diagnosis of air-space consolidation observed in chest radiographs includes bacterial pneumonia, pulmonary infarction, idiopathic OP, postradiation $\mathrm{OP}$, drug-induced $\mathrm{OP}$ or EP, idiopathic EP, bronchioloalveolar carcinoma, or lymphoma (5). In some cases, air-space consolidation has been seen as post-radiation OP in breast cancer patients during or following radiation therapy $(6,7)$. However, this patient did not receive radiation therapy. Given this patient's chemotherapy history and characteristic radiological findings, drug-induced pneumonitis with OP or EP type was first suspected. However, the finding that serum KL-6 levels were highly elevated in this patient was inconsistent with drug-induced pneumonitis with OP or EP type, idiopathic OP, or idiopathic EP since these diseases are unlikely to show increased serum levels of KL6 throughout their clinical course (8). The elevated serum 
(A)

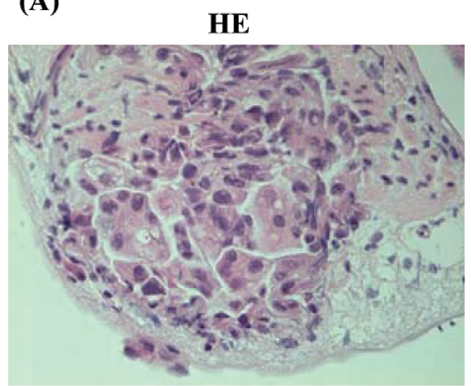

ER

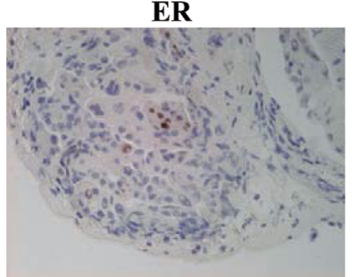

Her2



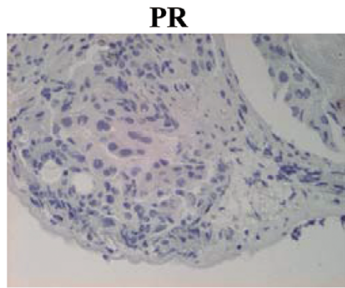

SP-A

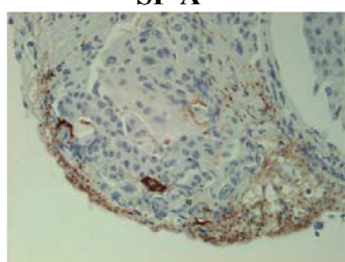

(B)


Her2



Figure 2. A transbronchial lung biopsy specimen from right lower lung lobe (A) was histologically consistent with a surgical specimen from original left breast cancer (B). Immunohistochemical studies with estrogen receptor (ER), progesterone receptor (PR), human epidermal growth factor receptor type 2 (Her2), and surfactant protein-A (SP-A) confirmed the tumor to be breast cancer and excluded the possibility of lung cancer. HE; Hematoxylin and Eosin Staining. Original magnification, $\times \mathbf{4 0 0}$.



Figure 3. Changes in air-space consolidation on chest CT were accompanied by changes in serum CEA levels before and after capecitabine monotherapy. Arrows indicate air bronchogram sign observed in the air-space consolidation.

KL-6 levels suggested the possible involvement of a disease other than OP or EP. Serum KL-6 levels are known to be increased in patients with adenocarcinoma including lung cancer, breast cancer, and pancreatic cancer (9). Serum KL6 levels are associated with tumor stage, distant metastasis, and relapse of breast cancer (10). Indeed, further histologi- cal and cytological analyses revealed that the air-space consolidation was caused by metastatic breast cancer in this patient. Since levels of tumor markers and KL-6 were highly elevated in the BAL fluid from the affected lung, increased serum levels of these markers might be partly due to increased production from metastatic breast cancer in the lung. 
Although KL-6 was not measured sequentially in the present case, changes in serum CEA levels were accompanied by radiographic changes on the chest CT (Fig. 3).

Metastases from adenocarcinoma may spread into the lung along the intact alveolar walls similar to a bronchioloalveolar carcinoma (11). This type of tumor expansion (lepidic growth) shows the radiological features of air-space consolidation mimicking bacterial pneumonia. Air-space consolidation is also seen in cases of metastases from gastrointestinal tract malignancies. In a retrospective study, six of 65 patients $(9.2 \%)$ with pulmonary metastases from an adenocarcinoma of the gastrointestinal tract had this pattern of metastasis (12). Breast, ovary, and pancreatic cancer may show a similar pattern of metastasis, but only a small number of cases has been reported $(3,13-15)$. Although the growth pattern of metastases from adenocarcinoma occasionally shows lepidic growth in histopathological studies, it is rarely sufficiently extensive to simulate confluent pneumonia-like opacity on chest radiography (2). The diagnosis of bronchioloalveolar carcinoma cannot be made with confidence if extrapulmonary adenocarcinoma has not been ruled out because both tumors can have similar histological features. In the present case, immunohistochemical studies were used to exclude the possibility of lung cancer and to confirm the origin to be breast cancer.

Metastatic or relapsed breast cancer is treated by chemotherapy and/or radiation therapy to improve the quality of life and to prolong survival. Since patients with Her2positive status show a poor prognosis, trastuzumab, a chimeric monoclonal antibody against Her2, is introduced for the treatment of Her2-positive metastatic breast cancer (16). Trastuzumab plus docetaxel combination chemotherapy shows superiority in terms of response rate, survival, time to disease progression, time to treatment failure, and duration of response compared to docetaxel monotherapy (17). Therefore, the patient was first treated by this regimen of chemotherapy, but the treatment ultimately failed. Capecitabine, an oral prodrug of 5-fluorouracil, is considered as a reference treatment for taxane-pretreated metastatic breast cancer. Several studies have shown that capecitabine monotherapy consistently produces clinical efficacy including survival with a favorable safety profile (18). We treated our patient with oral capecitabine monotherapy and the metastatic lesions in the lung were dramatically decreased in size following this regimen, but the effect of capecitabine on multiple hepatic and bone metastases was poor. The patient finally died of hepatic failure from multiple hepatic metastases of breast cancer 1 year later. The chemosensitivity of metastatic lung lesions seemed to be different from that of primary lesion or other metastatic lesions in this patient. Since cancer may consist of heterogeneous clones of cancer cells that metastasize to different sites, biological characteristics of cancer including chemosensitivity may be different from site to site. Indeed, expression levels of progesterone receptor, estrogen receptor, and Her2 were different between the primary lesion and the metastatic lung lesions.

In conclusion, breast cancer rarely causes pulmonary metastases presenting as air-space consolidation, but physicians should be aware of this unusual form of breast cancer metastasis.

\section{References}

1. Kreisman H, Wolkove N, Finkelstein HS, Cohen C, Margolese R, Frank H. Breast cancer and thoracic metastases: review of 119 patients. Thorax 38: 175-179, 1983.

2. Fraser RS, Müller NL, Colman N, Pare PD. Secondary neoplasm. In: Fraser and Paré's Diagnosis of Diseases of the Chest. 4th ed. Fraser RS, Müller NL, Colman N, Paré PD, Eds. WB Saunders, Philadelphia, 1999: 1381-1417.

3. Jung JI, Kim HH, Park SH, et al. Thoracic manifestations of breast cancer and its therapy. Radiographics 24: 1269-1285, 2004.

4. U.S. Cancer Statistics Working Group. United States Cancer Statistics: 2004 Incidence and Mortality. Atlanta (GA): Department of Health and Human Services, Centers for Disease Control and Prevention, and National Cancer Institute; 2007.

5. Fraser RS, Müller NL, Colman N, Paré PD. Table of differential diagnosis. In: Fraser and Paré's Diagnosis of Diseases of the Chest. 4th ed. Fraser RS, Müller NL, Colman N, Paré PD, Eds. WB Saunders, Philadelphia, 1999: 3077-3098.

6. Crestani B, Valeyre D, Roden S, et al. Bronchiolitis obliterans organizing pneumonia syndrome primed by radiation therapy to the breast. Am J Respir Crit Care Med 158: 1929-1935, 1998.

7. Miwa S, Morita S, Suda T, et al. The incidence and clinical characteristics of bronchiolitis obliterans organizing pneumonia syndrome after radiation therapy for breast cancer. Sarcoidosis Vasc Diffuse Lung Dis 21: 212-218, 2004.

8. Ohnishi H, Yokoyama A, Yasuhara Y, et al. Circulating KL-6 levels in patients with drug induced pneumonitis. Thorax 58: 872$875,2003$.
9. Kohno N, Akiyama M, Kyoizumi S, Hakoda M, Kobuke K, Yamakido M. Detection of soluble tumor-associated antigens in sera and effusions using novel monoclonal antibodies, KL-3 and KL-6, against lung adenocarcinoma. Jpn J Clin Oncol 18: 203216, 1988.

10. Ogawa $Y$, Ishikawa T, Ikeda $K$, et al. Evaluation of serum KL-6, a mucin-like glycoprotein, as a tumor marker for breast cancer. Clin Cancer Res 6: 4069-4072, 2000.

11. Seo JB, Im JG, Goo JM, Chung MJ, Kim MY. Atypical pulmonary metastases: spectrum of radiologic findings. Radiographics 21: 403-417, 2001.

12. Gaeta M, Volta S, Scribano E, Loria G, Vallone A, Pandolfo I. Air-space pattern in lung metastasis from adenocarcinoma of the GI tract. J Comput Assist Tomogr 20: 300-304, 1996.

13. Rosenblatt MB, Lisa JR, Collier F. Primary and metastatic bronciolo-alveolar carcinoma. Dis Chest 52: 147-152, 1967.

14. Foster CS. Mucus-secreting 'alveolar-cell' tumour of the lung: a histochemical comparison of tumours arising within and outside the lung. Histopathology 4: 567-577, 1980.

15. Steinke K, Süess K, Wiesner W. Pulmonary metastases from pancreatic adenocarcinoma mimicking bronchoalveolar carcinoma. Eur Radiol 10: 1683-1684, 2000.

16. Hatake K, Tokudome N, Ito Y. 5. Tanstuzumab treatment for breast cancer. Intern Med 46: 149-150, 2007.

17. Marty M, Cognetti F, Maraninchi D, et al. Randomized phase II trial of the efficacy and safety of trastuzumab combined with docetaxel in patients with human epidermal growth factor receptor 2- 
Inter Med 48: 727-731, 2009 DOI: 10.2169/internalmedicine.48.1849

positive metastatic breast cancer administered as first-line treatment: the M77001 study group. J Clin Oncol 23: 4265-4274, 2005.
18. Seidman AD, O'Shaughnessy J, Misset JL. Single-agent capecitabine: a reference treatment for taxane-pretreated metastatic breast cancer? Oncologist 7 Suppl 6: 20-28, 2002.

(C) 2009 The Japanese Society of Internal Medicine

http://www.naika.or.jp/imindex.html 